\title{
Analgesic Effect of Electroacupuncture on Postherpetic Neuralgia: A Trial Protocol for a Multicenter Randomized Controlled Trial
}

\author{
Qianyan Liu (1) - Xiaoliang Wu $\cdot$ Jing Guo $\cdot$ Jie Gao $\cdot$ Bingyang Liu • \\ Yuhang Wang $\cdot$ Minghui Xia $\cdot$ Lixia Pei $\cdot$ Jianhua Sun
}

Received: May 18, 2021 / Accepted: June 16, 2021 / Published online: July 12, 2021

(C) The Author(s) 2021

\section{ABSTRACT}

Introduction: The chronic neuropathic pain associated with postherpetic neuralgia (PHN) can last for several months or even many years, seriously affecting the affected person's work, sleep, mood, and daily life activities. In generaly, current treatments for PHN are at best limited and unsatisfactory, and adverse

Qianyan Liu, Xiaoliang Wu and Jing Guo contributed equally to this work.

Supplementary Information The online version contains supplementary material available at https:// doi.org/10.1007/s40122-021-00283-8.

Q. Liu - X. Wu · J. Guo - B. Liu · Y. Wang - L. Pei · J. Sun

Department of Acupuncture and Rehabilitation, Affiliated Hospital of Nanjing University of Chinese Medicine, Nanjing 210029, Jiangsu, China

Q. Liu

School of Medicine and Holistic Integrative

Medicine, Nanjing University of Chinese Medicine, Nanjing 210023, Jiangsu, China

J. Gao

Hezhuang Street Community Health Service Center, Hangzhou 311222, Zhejiang, China

M. Xia

Department of Acupuncture, Affiliated Hospital of Nantong University, Nantong 226001, Jiangsu, China reactions are common, especially in elderly patients. Electroacupuncture (EA) is widely used clinically to manage painful diseases, but there remains a lack of evidence to support the effectiveness of EA on PHN. This study is designed to assess the efficacy and safety of EA on PHN, and to provide evidence-based medical evidence for EA treatment of PHN.

\section{Q. Liu}

NMPA Key Laboratory for Research and Evaluation of Tissue Engineering Technology Products, Key Laboratory of Neuroregeneration of Jiangsu and Ministry of Education, Co-innovation Center of Neuroregeneration, Nantong University, Nantong 226001, Jiangsu, China

L. Pei $(\square) \cdot J$. Sun $(\square)$ Acupuncture and Moxibustion Disease Project Group of China Evidence-Based Medicine Center of Traditional Chinese Medicine, Nanjing 210029, Jiangsu, China e-mail: 11801758@qq.com

J. Sun

e-mail: jhsun@njucm.edu.cn 
Methods: This multicenter, prospective, randomized controlled trial will recruit 448 patients with PHN at seven clinical centers. Multicenter stratified variable block randomization will be used, and the eligible patients will be randomly allocated in a ratio of $1: 1$ to the EA group or sham EA group. The EA group will receive 4 weeks of EA treatment, given as 30-min treatment sessions, once daily, 5 times per week, for a total of 20 treatments; the sham EA group will receive sham EA treatment under the same conditions.

Planned Outcomes: The primary outcome measure is the 11-point Numeric Rating Scale pain score at week 4 . The secondary outcome measures, including mechanical pain threshold, pain area of PHN, average number of pain episodes, the short-form McGill Pain Questionnaire 2, Zoster Brief Pain Inventory, the Depression, Anxiety, and Positive Outlook Scale, Patient Global Impression of Change, safety of EA, and use of concomitant medications, among others. The primary analysis of the outcomes will be the mixed-effect model with repeated measurement between groups on an intent-to-treat population.

Trial Registration: Clinicaltrials.gov identifier: NCT04560361. Registered 23 September 2020 (https://clinicaltrials.gov/ct2/show/NCT045603 61?term=NCT04560361\&draw=2\&rank=1).

Keywords: Analgesic effect; Electroacupuncture; Postherpetic neuralgia; Randomized controlled trial; Trial protocol

\section{Key Summary Points}

\section{Why carry out this study?}

The chronic neuropathic pain associated with postherpetic neuralgia ( $\mathrm{PHN})$ severely interferes with the patient's activities of daily living, while the standard medications for the management of PHN can only offer limited clinical benefit, with long-term medications having the potential to cause many adverse reactions.

This trial aims to assess the efficacy and safety of electroacupuncture (EA) for the relief and removal of pain due to PHN.

What might be learned from the study?

The results of this study may provide evidence-based medical evidence for the use of EA to treat PHN, and contribute to current international understanding of the clinical relevance of EA in the treatment of PHN.

This study may provide high-quality current evidence for patients, clinicians and policy-makers seeking innovative and effective ways to treat PHN, thus potentially facilitating translation of EA treatment of PHN into clinical practice and relieve or eliminate the pain of individuals with PHN. 


\section{INTRODUCTION}

Postherpetic neuralgia (PHN) is the most frequent complication of herpes zoster (HZ) and is caused by reactivation of varicella zoster virus (VZV), usually manifest as the persistence or reappearance of extreme pain in the virus-infected area after the herpes scabs fall off and the skin lesions heal. The pain associated with PHN is commonly stubborn and intractable, falling into three broad categories: persistent spontaneous pain, paroxysmal pain, and evoked pain which is a pathologic amplification of responses to light touch and other harmless stimuli (mechanical allodynia) or harmful stimuli (mechanical hyperalgesia) [1]. A systematic review and comprehensive literature search reported that the best estimate of incidence rate for PHN is $28.2-42.0 / 100,000$ person-years and that the frequency and severity of PHN increases with age [2]. Although the number of people infected with $\mathrm{HZ}$ who progress to PHN may decrease in the future, PHN is currently an area of considerable clinical importance [3].

The chronic neuropathic pain associated with PHN can last for several months or even many years, leading to sleep disturbances, anxiety, irritability, depression, fatigue, weight loss, social isolation, withdrawal from employment, and even loss of an independent life style [4, 5]. Although PHN severely interferes with the patient's activities of daily living and significantly reduces functional status and health-related quality of life, among which the latter is potentially the most troubling aspect, it is a condition that is often challenging to treat. There is currently no specific therapy for PHN; consequently, treatment is basically based on symptom control [1]. Based on consensus, the expert panel recommends supplementing pain treatment with tricyclic antidepressants (TCAs) or anticonvulsant drugs to relieve PHN-related pain [6] and, if necessary, with opioids, topical treatments, and intrathecal steroids to treat the severity of pain [7]. However, pharmacotherapy can only slightly relieve the pain to a certain extent, and there are still many PHN patients who do not respond in a clinically effective manner to drug treatment. In addition, long- term use of gabapentin or pregabalin, and in particular of opioids, can cause many adverse reactions, such as dizziness, drowsiness, reduced cognitive focus, nausea, fatigue, constipation or tinnitus, among others [8]. For elderly patients who already have one or more underlying diseases, especially those with gastrointestinal diseases, long-term medications aggravate their conditions, causing stomach pain, bloating, diarrhea or other serious discomforts. Due to the at best limited and often unsatisfactory clinical benefits of currently available drugs on PHN [7], long-term medication is often accompanied by a relatively high risk of adverse effects, which is especially important in the elderly, and is also accompanied by high costs (i.e., for consultation, medicines and hospital treatments) [9]. Consequently, there is an extremely urgent need for a therapy for PHN that might be effective as an analgesic and have little or no adverse effects on physical health.

Acupuncture may be an effective treatment option for PHN. Several small studies have shown the clinical relevance of electroacupuncture (EA) in the management of PHN [10-12]; however, there is a lack of powerful evidence on the benefit of EA in PHN, leading to the effects of EA on PHN remaining uncertain. Thus, we designed the present multicenter randomized parallel controlled clinical trial of EA on PHN which is rigorously designed and have an appropriate sample size, aiming to evaluate the effectiveness and safety of EA in pain relieving and pain removing in the treatment of patients with PHN.

\section{METHODS}

This clinical trial protocol is formulated, implemented, and reported in accordance with the Standard Protocol Items: Recommendations for Interventional Trials (SPIRIT) 2013 guidance and Standards for Reporting Implementation Studies (StaRI) Statement for content [13-16].

\section{Study Design and Objectives}

The Analgesic Effect of Electroacupuncture on Postherpetic Neuralgia (AEEPN) study is a 
multicenter, prospective, parallel-arm, randomized, participant-blinded, sham EA-controlled clinical trial involving an estimated 448 patients with PHN, which follows the ethical guidelines of the Declaration of Helsinki and the Quality Management Practices for Clinical Trials of Medical Devices. The objective of this study is to examine the effectiveness and safety of EA for the treatment of PHN. The study duration (see Fig. 1) is about 10 weeks, which includes a washout period ( $\geq 7$ days), a screening observation period (3-7 days), a treatment period (4 weeks), and a follow-up period (1 month). The ethics committees of all seven participating hospitals approved the study protocol (the full trial protocol is available in the Electronic Supplementary Material [ESM]), and all participating patients will provide written informed consent before entering the study (see flow diagram of patient enrollment in Fig. 2).

\section{Study Setting}

The AEEPN trial will recruit patients with PHN from the outpatient and inpatient units of departments of acupuncture at seven clinical centers located in six different regions in China. All clinical centers are comprehensive hospitals integrating medical and health, teaching, and scientific research, and all are tertiary grade A or B hospitals equipped with high-level medical technology and complete scientific research equipment. Thus, they can provide patients with highly professional medical services and ensure the implementation of AEEPN. The primary sponsor will collect and manage information data from sub-centers through the case report form (CRF) and electronic data capture (EDC) system. The independent Data Safety Monitoring Board will monitor security periodically throughout the study period.

\section{Sample Selection/Eligibility Criteria}

Acupuncturists responsible for acupuncture treatments in the seven clinical centers must have a qualification certificate and independently undertake clinical treatment for $>$ 2 years. All participants will fulfill the diagnostic criteria for PHN according to the European consensus-based (S2k) Guideline $[17,18]$, namely, have a clear history of acute $\mathrm{HZ}$ and persistence of pain for $>90$ days after the onset of erythematous macules and rash. Auxiliary indications for PHN are: lesions are usually accompanied by paroxysmal, persistent, or evoked pain, sensory abnormalities, and hyperalgesia, and are characteristically distributed according to the innervation area; lesions may be accompanied by manifestations of autonomic nervous dysfunction, such as hyperhidrosis; local skin can manifest residual scars or pigmentation; and changes in the patient's ability to function in activities of daily living, which may/may not be accompanied by sleep disorders, anxiety, low mood, and depression.

The main inclusion criteria for the AEEPN trial are: man or woman; age between 45 and 75 years; meeting the diagnostic criteria for PHN; diagnosis of moderate or higher level of pain during the screening observation period (daily 11-point Numeric Rating Scale [NRS-11] pain scores were collected during the observation period, and the average pain score was $\geq 4$ ); and willingness to participate in the AEEPN trial and to sign the informed consent form (ICF).

Patients meeting the following exclusion criteria will be excluded from the study: (1) patients who are currently receiving, or have received more than one "permitted concomitant medication" or any "prohibited concomitant medications and treatments" for the treatment of PHN at least 14 days before the screening observation period, and are unwilling to undergo a washout period ( $\geq 7$ days); (2) patients with serious safety issues during the washout period or observation period; (3) patients with serious uncontrolled medical conditions; (4) patients with pain unrelated to PHN; (5) patients who have undergone neurological intervention or other surgical operations on PHN, such as interventions for selective nerve damage or radiofrequency thermocoagulation damage (all information on the inclusion and exclusion criteria is shown in the ESM). 


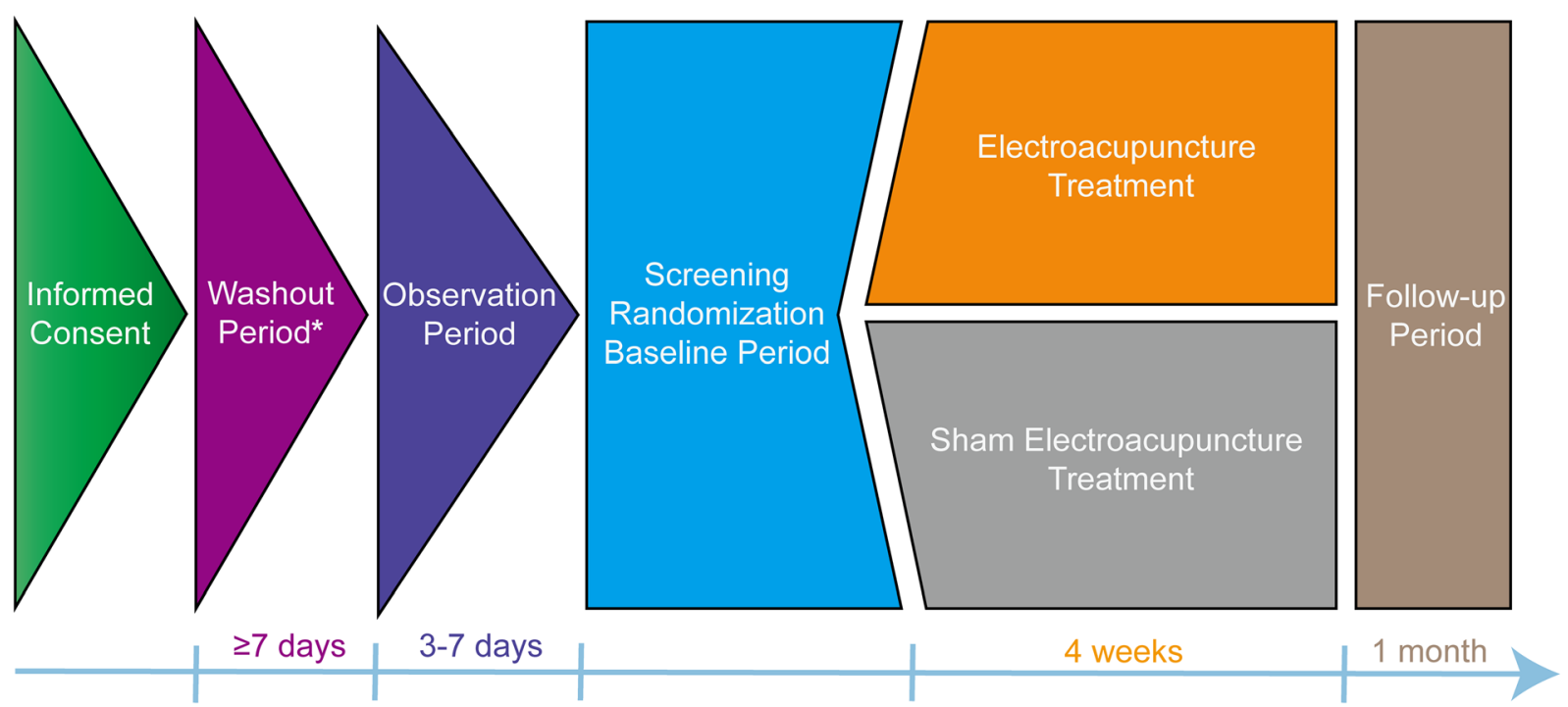

Fig. 1 Study design. Screening and randomization will be performed based on an average daily pain score of $\geq 4$, assessed using the 11-point Numeric Rating Scale (NRS11) during the observation period. Electroacupuncture treatment arms are shown in orange and gray. Patients will be followed up for 1 month after treatment. Asterisk: The washout period is required only for specific patients, namely, those currently receiving or having received $>1$

\section{Interventions}

All participants will receive lifestyle interventions as basic treatment. These include recommending a reasonable diet for patients; promoting patient knowledge on a healthy diet in terms of low salt, low fat and low sugar intake; identifying patients' concerns; providing help to patients for the relief of stress factors and reduction of avoidance behavior; establishing a positive doctor-patient relationship; reviewing patients' drug dosage, quality of life and ability to carry out activities of daily living; assessing patients' anxiety and depression; and providing healthy lifestyle education.

The EA therapy program has been developed by the consensus reached by acupuncture experts based on previous studies of EA for PHN. The reader is referred to the National Standard of the People's Republic of China "Acupoint Name and Location" (GB/T 12346-2006) for acupoint location. This study will use Hwato brand disposable acupuncture needles (size 0.30 permitted concomitant medication or any prohibited concomitant medications and treatments listed in sectionConcomitant Medications for the treatment of postherpetic neuralgia within 14 days; for these patients, a washout period of $>7$ days (depending on the half-life of the medications) is required after informed consent

$\times 40 \mathrm{~mm}$ ), placebo blunt needles (size $0.40 \times$ $13 \mathrm{~mm}$ ), insulating adhesive pads (Fig. $3 \mathrm{a}-\mathrm{c}$ ), and SDZ-V EA apparatuses (Suzhou Medical Instrument, China).

Participants randomly assigned to the EA group will receive acupuncture at ipsilateral (PHN-affected side) Zhigou (SJ6; located 3 cun [four finger-widths] above the transverse crease on the back of the wrist, between the ulna and radius), Yanglingquan (GB34, located in the anterior inferior depression of the fibular head), and Ashi points (every 2- to $3-\mathrm{cm}$ interval around the painful area of $\mathrm{PHN}$ is used as an acupuncture point) (Fig. $3 \mathrm{~d}, \mathrm{~d}^{\prime}$ ). Participants take the appropriate position on the treatment bed according to the location of PHN. After routine skin disinfection, the sterile insulating adhesive pads are placed on the ipsilateral Zhigou, Yanglingquan and Ashi points. The acupuncture needles are then inserted vertically into the skin at the Zhigou and Yanglingquan points through the pads for approximately 15-20 mm, accompanied with slight, equal 


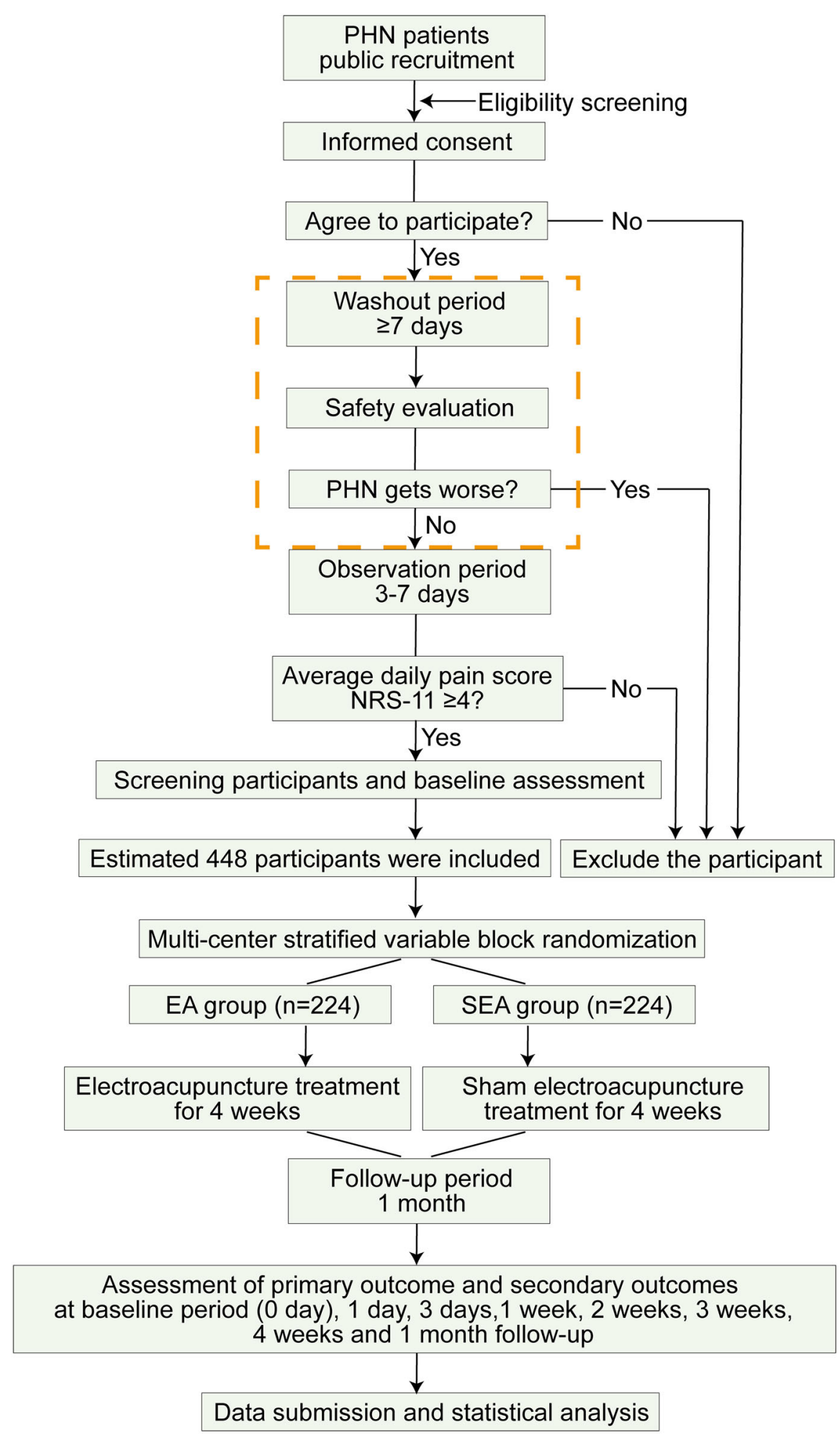

Fig. 2 Flow diagram of patient enrollment. The yellow dotted box indicates that patients who meet the standards of concomitant medications do not require a washout period and can directly enter the observation period. $E A$ Electroacupuncture, $P H N$ postherpetic neuralgia, $S E A$ sham electroacupuncture 
manipulations of twirling and lifting to reach the sensation of deqi (known as soreness, numbness, distention, heaviness, or another sensation, which are considered to indicate effective acupuncture) (Fig. 3e). Acupuncture needles are also inserted obliquely (at an angle of $30^{\circ}-45^{\circ}$ ) into the skin at the Ashi points through the pads for approximately $10 \mathrm{~mm}$ (Fig. 3f), In all cases, the needle point is towards the painful area of PHN. The paired poles of the EA apparatus are connected to the needle handles for the ipsilateral Zhigou and Yanglingquan points; for Ashi points, the paired poles are connected to the head and tail acupoints of the long axis of the painful area. The waveform of EA stimulation is a continuous wave with a frequency of $2 \mathrm{~Hz}$ and a current intensity of 1-5 mA (causing a mild tremor of the skin around the acupoints without pain), which will continue for the 30-duration of each EA treatment session. All patients will receive EA treatment once per day, 5 times a week for 4 consecutive weeks (20 treatments in total).

Participants randomly assigned to the SEA group will received SEA treatment by using placebo blunt needles at the same acupoints. After disinfecting the skin and placing the sterile insulating adhesive pads on ipsilateral Zhigou, Yanglingquan and Ashi points, placebo blunt needles are inserted through the pads and reach the insulating adhesive layer (Fig. $3 g$, h), causing the participants to feel the needle resistance (a sensation of needle insertion). Other procedures, electrode placements, parameters of EA apparatus, and treatment settings are the same as in the EA group, but with no skin penetration or electricity output.

\section{Potential Risks/Harm and Trial Termination}

Both the EA and SEA treatments will be in compliance with the clinical practice of acupuncture, but there is still a risk of adverse events (AEs) caused by acupuncture, including acupuncture syncope, broken needles, unbearable acupuncture pain (NRS $\geq 8$ points), local infection, subcutaneous congestion or hematoma, other discomforts after acupuncture (symptoms such as post-acupuncture pain, nausea, vomiting, palpitations, dizziness, and headache that last for $>1 \mathrm{~h}$ ), and other unforeseen AEs. Any AEs and serious AEs, including the severity, treatment measures, and the outcome of the signs/symptoms of concomitant disease, will be assessed and documented during the visits. Appropriate and timely medical treatments will be provided by the researchers according to the condition; if necessary, the procedure will be suspended to ensure the safety of the participants. During the study period, should participants experience any potential exacerbation of symptoms or develop another emerging serious disease affecting the observation of the trial, they will be provided with emergency treatment and the participant will be excluded from the trial according to the physician's assessment of the condition.

\section{Adherence}

No participant will not be liable for any costs during the treatment period (including registration fees, EA treatment fees and other medical expenses); all costs are sponsored by the project funding. Participants will be provided with a detailed explanation of the purpose of the trial, the necessity for the observation indicators, and the EA treatment and related examinations. The consent and cooperation of all participants will be obtained. Based on the principle of voluntary participation, an ICF will be explained to and subsequently signed by each participant. Throughout the trial, the participants will be held separately during treatment to prevent communication. The participants' demographic information will be recorded in detail for follow-up. Participants will be provided with a good consultation environment, medical services, and disease assessment by professionals throughout the study period, and the establishment of a good doctor-patient relationship will be actively worked on to encourage patients to complete the entire study. 


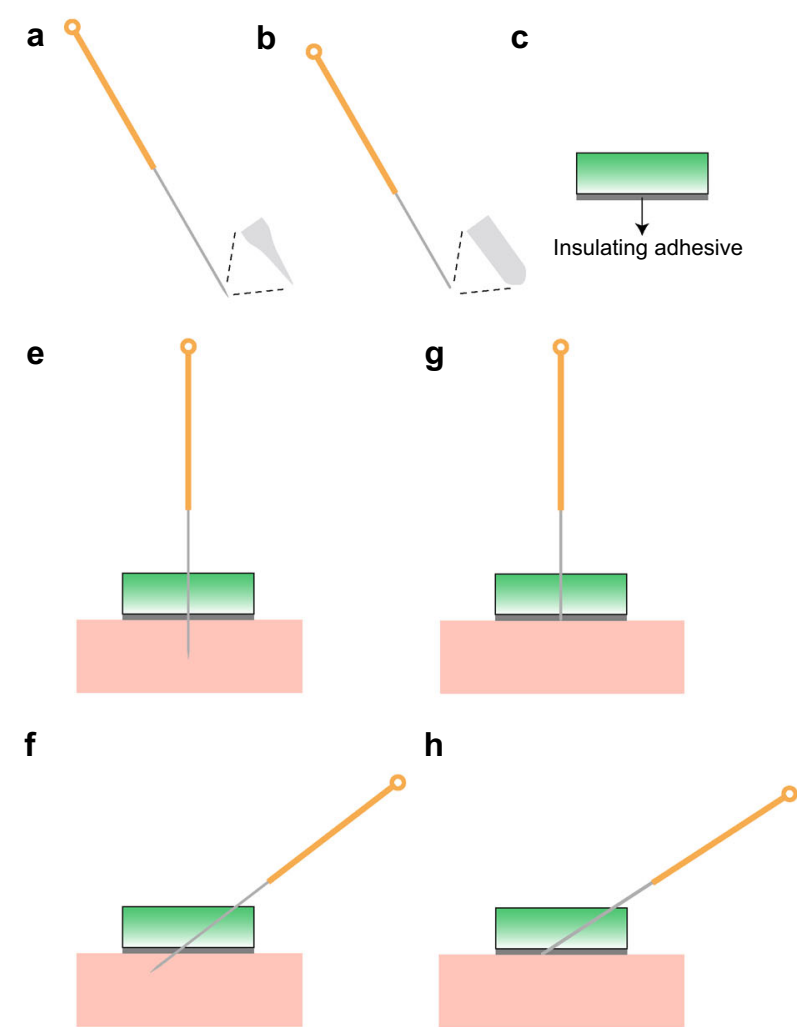

Fig. 3 Location of acupoints in the study, and acupuncture procedure for the EA and SEA groups. a Disposable acupuncture needles (size $0.30 \times 40 \mathrm{~mm}$ ). b Placebo blunt needles (size $0.40 \times 13 \mathrm{~mm}$ ). c Insulating adhesive pads. d Location of ipsilateral (PHN-affected side) acupoints in the study. For example, for PHN in the left lumbar, choose the Zhigou (SJ6), Yanglingquan (GB34), and Ashi points on the left side for the EA treatment; refer to the same location on the right. $\mathbf{d}^{\prime}$ Enlarged view of the location of Ashi points. Every 2- to $3-\mathrm{cm}$ interval around the painful area of PHN is used as an acupuncture point, with the

\section{Concomitant Medications}

During the first visit, participants will provide their medication usage during the previous 2 weeks, including drug name (or other therapy name), indications, dosage unit, frequency of administration, route of administration, and start and end time of medication. This information will be recorded.

Participants will be allowed to take only one of the following medications to treat PHN if they take a stable dose level for PHN at least 14 days before the screening period: any

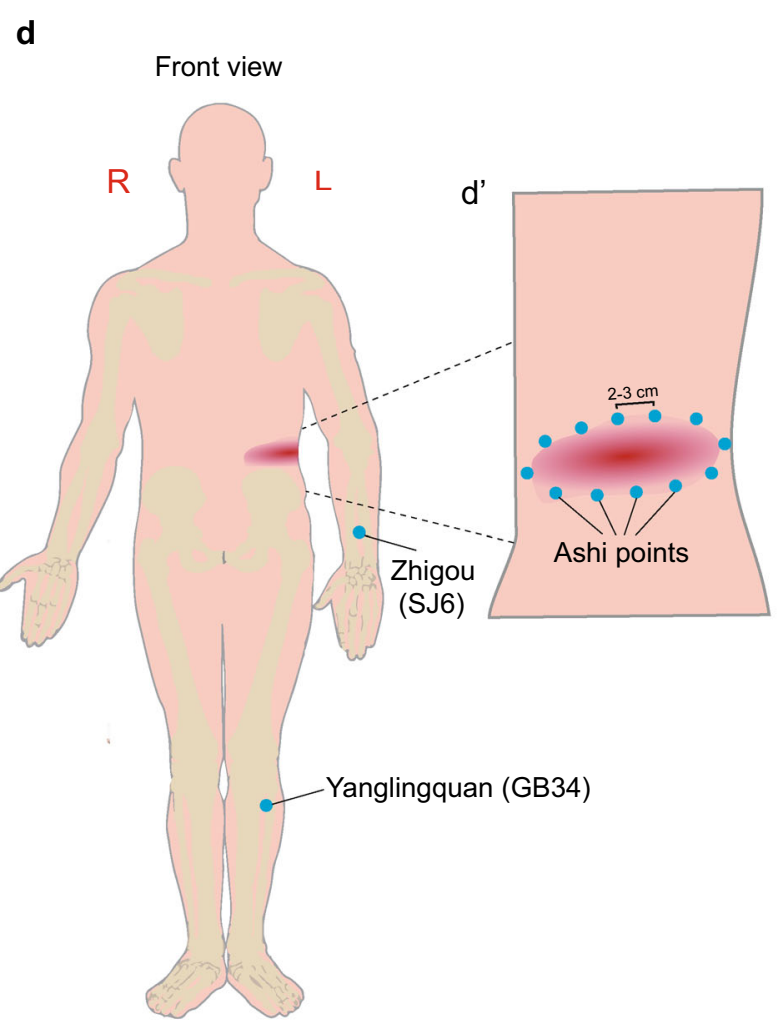

needle pointed towards the painful area. e Operating diagram of inserting needle vertically into the Zhigou and Yanglingquan points in the EA group. f Operating diagram of inserting needle obliquely (at an angle of $30^{\circ}-45^{\circ}$ ) into the Ashi points in the EA group. $\mathbf{g}$ Operating diagram of inserting needle vertically into the Zhigou and Yanglingquan points in the SEA group. $\mathbf{h}$ Operating diagram of inserting needle obliquely (at an angle of $30^{\circ}-45^{\circ}$ ) into the Ashi points in the SEA group

anticonvulsant drugs (e.g., pregabalin, gabapentin); any one of serotonin-norepinephrine reuptake inhibitors (SNRIs) (e.g., venlafaxine, duloxetine); any TCA (e.g., amitriptyline, nortriptyline); any opioid analgesic (e.g., tramadol, codeine); or any one of oral non-steroidal antiinflammatory drugs (NSAIDs) (e.g., ibuprofen, celecoxib). Thereafter, the participants should maintain the medication regimen during the entire study period unless there are safety issues during the observation period. If a participant wants to adjust his medication regimen to apply to his or her doctor, the participant can only 
adjust the medication plan after the doctor has assessed the specific condition. Participants will be allowed to take a stable dose of the following drugs for the treatment of underlying diseases throughout the study: stable dose of diuretics, calcium antagonists or related drugs for the treatment of hypertension; the selected 5-SNRI for depression; other drugs not included above and not related to the treatment of PHN, among others.

The main prohibited concomitant medications for the AEEPN trial are as follows: any topical medication for PHN, including 5\% lidocaine patch, lidocaine ointment, capsaicin patch, and any other topical drug preparations for the treatment of PHN; any skeletal muscle relaxant; any antipsychotics or neuroleptic medications; and any other medication for pain relief not included above, among others. During the study period, any neuromodulation techniques, nerve block, and other forms of analgesic therapy that may confuse the evaluation of PHN is prohibited (detailed information on permitted and prohibited concomitant medications is available in the ESM).

Participants will be allowed to take oral paracetamol/acetaminophen (up to $2 \mathrm{~g} /$ day, as needed) as rescue medication for unacceptable pain. The use of rescue medication must be promptly recorded in the CRF and entered into the EDC system, and all participants are discouraged from receiving any prohibited medications or treatments throughout the trial; if received, they are asked to document all.

\section{Measurements and Planned Outcomes}

Patients with PHN endure long-term pain, and their sleep quality, mental state, daily activities, and work and living activities are seriously affected. Hence, our outcomes are patient-reported measures of pain and quality of life. The primary outcome is the NRS- $11[19,20]$ pain score at week 4 . The NRS- 11 score ranges from 0 (no pain) to 10 (worst possible pain), with 1-3 points representing mild pain, 4-6 points representing moderate pain, and 7-10 points representing severe pain. The NRS-11 is widely used; it demonstrates good reliability, achieves higher patient compliance, and is easy to use and more sensitive than other pain measures [21].

The secondary outcome measures for the trial include the visual analogue scale (VAS) $[19,20,22]$, verbal rating scale (VRS) $[19,21]$, mechanical pain threshold (MPT) [23-27], pain area of PHN (PAP) [28-30], average number of pain episodes (ANPE) [31], average duration of each pain episode (ADEPE), short-form McGill Pain Questionnaire 2 (SF-MPQ-2) [32-37], Zoster Brief Pain Inventory (ZBPI) [38], Depression, Anxiety and Positive Outlook Scale (DAPOS) $[39,40]$, Patient Global Impression of Change (PGIC) [40, 41], safety of EA, and use of concomitant medications. The VAS score ranges from 0 (no pain) to $10 \mathrm{~cm}$ (worst imaginable pain), and is a horizontal line without verbal descriptors or numbers at intermediate points [20]. The VAS is chosen because of its simplicity, adaptability to a broad range of populations and, more importantly, it has good visual properties. The VRS is a measure from 0 to 3 according to the patient's chief complaint and assesses the interference of pain on sleep. In addition, it has a wide range of VAS and NRS scores within each VRS category [19] and has a good correlation with NRS. For testing mechanical sensitivity of the PHN patient's painful skin, we stimulate the center of the pain area (avoid scars and injured areas) with a series of von Frey hairs with logarithmically increasing stiffness (0.008-300 g; 514000-20C, DanMic Global, LLC, San Jose, CA, USA) [25]. The MPT is chosen because of it is a relatively objective outcome measure for assessing pain changes and skin mechanical sensitivity. The PAP is calculated by the researcher based on the 45 Body Areas Rating Scale (BARS-45) [28]. The PAP, ANPE, ADEPE, and ZBPI are questionnaires specially formulated for $\mathrm{HZ}$ to assess the range, frequency, duration, and degree of pain and its impact on a patient's work life, interpersonal relationships, and sleep [38] that take approximately 5 min to complete. The SF-MPQ-2 consists of 22 items, which combine continuous pain (6 items), intermittent pain (6 items), neuropathic pain (6 items), and affective descriptors (4 items). The SF-MPQ-2 has excellent reliability and validity, and can provide a 
single measure of the main sensory and affective symptoms of neuropathic pain [33, 37]. DAPOS is a reliable and brief questionnaire that has been particularly developed to assess mood in people with pain; it takes about $3 \mathrm{~min}$ to complete. The PGIC is a 5-point single item [41] for assessing the improvement, stability or worsening of PHN from baseline to 4 weeks after treatment. The safety of EA will be evaluated after each treatment (see section Potential Risks/Harm and Trial Termination). The percentage of patients using concomitant medications and the changes in dosage and frequency of concomitant medications (including rescue medication) will be analyzed after the completion of the trial. The time points for each outcome and the time schedule of enrollment, screening, washouts, observation, and treatment for participants are available (see Fig. 4).

\section{Sample Size and Recruitment}

According to previous studies, it is anticipated that a difference in primary outcome (NRS-11) between the EA group and SEA group is 1.2 and the combined standard deviation (SD) of the two groups is 3.5 . To achieve $90 \%$ statistical power and keep the type I error rate to $<0.05$, we calculated, using PASS version 15 (NCSS, LLC. Kaysville, UT, USA), that a sample of 448 participants (224 participants in each group) will be required to detect a two-sided significant difference between the two groups and compensate for a dropout rate of $20 \%$. All participants are equally distributed among seven clinical centers, and 64 participants per center will be recruited. Patients with PHN will be recruited from hospitals through posters, public social networking platform (Sina Weibo, WeChat public account), and advertisements.

\section{Allocation and Blinding}

Eligible patients will be randomly allocated in a ratio of 1:1 to the EA or SEA group by EDC's central randomization system. The randomization sequence in this study uses multicenter stratified variable block randomization, and the block size of each center is randomly set to 4,6 , and 8. Considering that the number of patients in each stratum fluctuates due to block variation, 21 stratums are preset at random and take the first seven groups with the number of patients equal to 64 as the final grouping. The randomization sequence is generated and validated by an independent statistician using $\mathrm{R}$ software (R Foundation for Statistical Computing, Vienna, Austria), then imported into EDC's central randomization system and kept by an administrator who does not participate in this trial. The central randomization system has set strict personnel permission levels, and no one but the highest-level system administrator has the right to view the randomization scheme. After participants meet all the inclusion criteria and complete the baseline assessment, an acupuncturist will $\log$ in to the central randomization system to apply for random numbers which will then be displayed, and the patients will receive the corresponding treatment. The clinical research coordinators (CRCs) will be responsible for enrolling participants, obtaining informed consent, and recording the CRF. Patients and CRCs (i.e., outcome assessors, data recorders, data entry clerks, and statistical analysts) will be blinded to the group assignment. Due to the nature of the intervention, acupuncturists will not be blinded to the treatment.

\section{Data Collection, Management, Monitoring and Auditing}

Data collection and analysis, quality assurance, and trial conduct and implementation will follow Good Clinical Practice (GCP) guidelines [42]. Before the AEEPN trial is officially initiated, the research group will hold a specialized clinical training meeting for all clinical researchers (including acupuncturists, outcome assessors, data recorders, data entry clerks, research nurses, quality controllers, and other related researchers) to achieve uniformity in terms of interpretation and training on the study implementation scheme, standard operation procedure (SOP), filling in of CRF, EDC system use, and electronic data entry. The aim is that researchers in each clinical center will be 


\begin{tabular}{|c|c|c|c|c|c|c|c|c|c|c|}
\hline \multirow{3}{*}{$\begin{array}{l}\text { Study period } \\
\text { Visit } \\
\text { Timepoint }\end{array}$} & \multirow{3}{*}{$\begin{array}{l}\text { Washout } \\
1 \\
\leq-1 \text { weeks }\end{array}$} & \multirow{3}{*}{$\begin{array}{l}\text { Observation } \\
2 \\
-3 \text { to }-7 \text { days }\end{array}$} & \multirow{3}{*}{$\begin{array}{c}\text { Baseline } \\
3 \\
0 \text { day }\end{array}$} & \multicolumn{6}{|c|}{ Treatment (5 times a week) } & \multirow{3}{*}{$\begin{array}{c}\text { Follow-up } \\
24 \\
1 \text { month }\end{array}$} \\
\hline & & & & 4 & 6 & 8 & 13 & 18 & 23 & \\
\hline & & & & 1 day & 3 days & 1 week & 2 weeks & 3 weeks & 4 weeks & \\
\hline \multicolumn{11}{|l|}{ Patients } \\
\hline Informed consent & $\mathrm{X}$ & & & & & & & & & \\
\hline Screening & & & $\mathrm{x}$ & & & & & & & \\
\hline Demographic data & & & $\mathrm{X}$ & & & & & & & \\
\hline History of PHN & & & $x$ & & & & & & & \\
\hline Basic disease histor & & & $x$ & & & & & & & \\
\hline Randomization & & & $\mathrm{x}$ & & & & & & & \\
\hline \multicolumn{11}{|l|}{ Primary outcome } \\
\hline NRS-11 & & & $\mathrm{x}$ & $\mathrm{x}$ & $\mathrm{x}$ & $\mathrm{x}$ & $\mathrm{x}$ & $\mathrm{X}$ & $\mathrm{X}$ & $\mathrm{X}$ \\
\hline \multicolumn{11}{|l|}{ Second outcome } \\
\hline Visual analogue sca & ale & & $\mathrm{x}$ & $\mathrm{x}$ & $\mathrm{X}$ & $\mathrm{x}$ & $x$ & $\mathrm{x}$ & $\mathrm{x}$ & $\mathrm{X}$ \\
\hline Verbal rating scale & & & $x$ & $x$ & $x$ & $\mathrm{x}$ & $x$ & $x$ & $x$ & $x$ \\
\hline$\overline{\mathrm{MPT}}$ & & & $x$ & $\mathrm{x}$ & $\mathrm{X}$ & $\mathrm{X}$ & $\mathrm{X}$ & $\mathrm{X}$ & $\mathrm{X}$ & $\mathrm{X}$ \\
\hline Pain area of $\mathrm{PHN}$ & & & $\mathrm{x}$ & $\mathrm{x}$ & $\mathrm{x}$ & $\mathrm{x}$ & $\mathrm{x}$ & $\mathrm{x}$ & $x$ & $x$ \\
\hline ANPE & & & $x$ & $x$ & $x$ & $x$ & $x$ & $\mathrm{x}$ & $x$ & $x$ \\
\hline ADEPE & & & $\mathrm{X}$ & $\mathrm{X}$ & $x$ & $x$ & $x$ & $x$ & $\mathrm{X}$ & $x$ \\
\hline SF-MPQ-2 & & & $x$ & & & & & & $x$ & $x$ \\
\hline ZBPI & & & $x$ & & & & & & $x$ & $x$ \\
\hline DAPOS & & & $\mathrm{X}$ & & & & & & $\mathrm{x}$ & $\mathrm{X}$ \\
\hline PGIC & & & & & & & & & $x$ & $\mathrm{X}$ \\
\hline SEA & & & & $x$ & $\mathrm{x}$ & $\mathrm{x}$ & $x$ & $\mathrm{x}$ & $x$ & \\
\hline Concomitant medicatic & tion & & & $x$ & $x$ & $x$ & $x$ & $x$ & $\mathrm{X}$ & $\mathrm{X}$ \\
\hline Assessment of blindin & & & & $x$ & $x$ & $x$ & $x$ & $x$ & $x$ & \\
\hline Adherence & & & & $\mathrm{X}$ & $\mathrm{X}$ & $x$ & $\mathrm{X}$ & $x$ & $\mathrm{X}$ & \\
\hline Adverse events & & & & $x$ & $x$ & $x$ & $x$ & $x$ & $x$ & $x$ \\
\hline
\end{tabular}

Fig. 4 Schedule of study procedures. MPT Mechanical pain threshold, $A N P E$ average number of pain episodes, $A D E P E$ average duration of each pain episode, $S F-M P Q-2$ short-form McGill Pain Questionnaire 2, ZBPI Zoster Brief Pain Inventory, APOS Depression, Anxiety and

familiar with the study protocol and SOP, thus improving the internal observation consistency of researchers.

All CRFs for each participant will be filled in promptly by researchers in each clinical center, including the assessment scales, questionnaires, treatment compliance, concomitant medication use, AEs, etc. For any evidence-based correction of CRF, only lines can be drawn. Any marginal notes on modified data will be signed and dated by the researcher, and the original records will not be erased or overwritten. All original documents and CRFs will be stored in the clinical study office. In addition, all data will be accurately double-entered into the EDC system, and the electronic CRF will be identical to the paper CRF. Once the data entry is completed, the database will automatically perform
Positive Outlook Scale, PGIC Patient Global Impression of Change, $S E A$ safety of electroacupuncture

consistency checks, missing value checks, and value range and logic checks. If any inconsistencies or missing data are found, the data will be re-checked and corrected based on the original documents and CRFs. The contents, time, and operators of all operations will be recorded by the EDC system to ensure the accuracy, completeness, and traceability of the research data entry. When $50 \%$ of the participants' data is entered into the EDC system, we will perform an interim analysis on the primary outcome, whichever comes first. This will be performed by an independent statistician, and the results will inform us whether the intervention has been proved to be effective (beyond reasonable doubt). Subsequently, we will decide whether (if necessary) to modify the study or terminate it prematurely. When all data are entered in the 
EDC system, $10 \%$ of the eCRF will be randomly selected for manual inspection, and the database will be locked and sent to the statisticians for further statistical analysis after all data inspection is completed and updated. Finally, we will submit the end of trial notification and final report to the Ethics Committee of each of the seven participating clinical centers, the Sponsor, and ClinicalTrials.gov.

\section{Data Analysis}

All statistical analysis (i.e., baseline characteristics, clinical outcomes) will be based on the intention-to-treat (ITT) population of all randomly assigned patients and performed using SAS software, version 9.4 (SAS Institute Inc. Cary, NC, USA). Missing data of withdrawn participants will be imputed using the multiple imputation method. Descriptive statistics will be presented as mean $\pm S D$, frequency, numbers or percentages when appropriate. The primary analysis will be the mixed-effect model with repeated measurement (MMRM) of changes between groups at 1 day, 3 days, 1 week, 2 weeks, 3 weeks, 4 weeks, and 1 month of follow-up in primary and secondary outcomes with covariates: baseline mean pain intensity, area and duration of PHN, onset time, age, sex, etc. For other supportive analysis, we will fit a multiple logistic regression model to compare the proportion of participants responding in each group, adjusting for other factors, such as randomization stratification of the seven clinical centers and use of concomitant medications. In addition, all numerical variables when normally distributed will be compared using the Student's $t$ test (homogeneity test of variance will be performed between groups, and the Satterthwaite method will be used to correct the Student's $t$-test when the variance is uneven), and the Wilcoxon rank sum test will be used when data are not normally distributed. Categorical variables will be compared using the chisquared test or the Fisher's exact test as appropriate. All $P$ values will be from two-sided tests and $P<0.05$ will be considered statistically significant.

\section{Informed Consent}

Researchers in each research center will introduce the study in detail to patients so that they can fully understand the potential risks and benefits of participating in this study. The researchers will obtain written informed consent (see ESM) from all participants recruited to the study. Participants will also be informed that they are not obligated to participate in the study and are free to withdraw at any time without any negative impact on their future medical treatment.

\section{Confidentiality}

Personally identifiable information of all participants, such as names, gender, among others, will be replaced by codes or numbers throughout the study and will remain confidential at all times, with only the relevant doctors aware of the patient's personal information. Any identifiable information will be stored separately from the study data, and any public reports on the results of this study will not reveal any identifiable information about the patient. The patient's medical records will be kept in the hospital. Researchers, research authorities, and ethics committees will be allowed to access the medical records to check whether the study is performed appropriately. If the patient signs the ICF, it means that he or she consents to be consulted by the above-mentioned personnel.

\section{DISCUSSION}

In recent years, many authoritative journals have reported that acupuncture plays an active role in the treatment of pain-related diseases [44-48]. The results of one large clinical trial suggest that acupuncture might significantly reduce pain in women with breast cancer and help patients adhere to life-saving cancer treatments, potentially improving survival rates [49]. A randomized clinical trial showed that adjunctive therapy with acupuncture had a significant effect in alleviating angina within 16 weeks [48]. Another individual patient data 
meta-analysis of only high-quality trials showed that acupuncture is associated with reductions of chronic pain [45]. At the present time, despite current treatments for PHN only offering limited benefit and being commonly associated with adverse reactions, and EA being widely used clinically to manage painful diseases, there remains a lack of related evidence of EA for PHN. The AEEPN trial is designed to assess the effectiveness and safety of EA on PHN in terms of relief and removal of pain and to provide evidence-based medical evidence for EA treatment of PHN, thereby improving the clinical knowledge of PHN treatment options in today's patient population. It will recruit 448 patients with PHN in seven clinical centers with the aim to assess whether EA can effectively reduce the degree, area, or frequency of pain in PHN patients, and whether EA can improve the impact of PHN on sleep, work and daily living activities. In general, the results of our study will contribute to today's international understanding of the clinical relevance and effectiveness of EA and PHN, thus introducing the use of EA as a potential treatment option with minimal side effect for PHN patients, aimed at relieving their neuralgia and skin sensitivity and improving their quality of life and work participation.

\section{STRENGTHS AND LIMITATIONS}

The AEEPN trial has several strengths. It is the first randomized controlled clinical trial of EA in PHN patients with the aim to prove the potential analgesic effect of EA in this setting. To our knowledge, this will be the largest multicenter study on EA treatment of PHN so far; hence it will be expected to provide high-quality and upto-date evidence for patients, clinicians, and policy-makers seeking innovative and effective ways to treat PHN. Moreover, unlike the control group used in many current studies [43, 50, 51], which use shallow acupuncture, this study used a new sham acupuncture technique for the first time, which causes patients to feel acupuncture but the needles do not penetrate the skin. This experimental design of sham EA is more rigorous for controlled studies and more convincing for verifying the true effect of EA. Compared with the current clinically widely used drugs for the treatment of PHN, such as gabapentin and pregabalin, the special strength of EA is that it can be directly applied to the painful area; the electrical stimulation applied can excite nerve endings on the skin surface of the painful area, thus producing analgesic effects. EA therapy is safe and has few side effects, such as possible slight needling pain, avoids damage to the stomach, liver, and kidney possibly caused by oral medicine, and is an especially friendly treatment for elderly patients with weakened immunity.

However, with respect to limitations of the AEEPN trial, we must note the methodological limitation that acupuncturists will not be blinded to the treatment allocation due to the nature of the intervention. Patients must undergo a washout period of longer than 1 week (if needed) and an observation period of 3-7 days, which increases the possibility that PHN might be exacerbated before the patient is included in the trial. In addition, patients need to come to the clinic for EA treatment five times a week, which is not as convenient as taking drugs at home.

\section{ETHICS AND DISSEMINATION}

The AEEPN trial will be conducted in accordance with the principles of the Declaration of Helsinki, the Quality Management Practices for Clinical Trials of Medical Devices, and International Code of Ethics for Research on Human Health. It has been reviewed and approved by the Ethics Committee of all study centers (Jiangsu Province Hospital of Chinese Medicine [Primary sponsor]; Approval No. 2020NL103-03), Nanjing Hospital of Chinese Medicine Affiliated to Nanjing University of Chinese Medicine (Endorsed and shared the ethics approval documents of the primary sponsor), Wuxi Hospital of Traditional Chinese Medicine (Approval No. HX2020082402), Nantong Hospital of Traditional Chinese Medicine (Approval No. [2020] 008), Shuyang Hospital of Traditional Chinese Medicine (Approval No. 20200824-001), Huai'an Hospital of Traditional 
Chinese Medicine (Endorsed and shared the ethics approval documents of the primary sponsor), and Lianyungang Hospital of Traditional Chinese Medicine (Approval No. 2021-KY-05). The researchers will follow GCP principles and the approved protocol to implement clinical research and protect the health and rights of each patient. Consent to participate in the study will be obtained using an written informed consent (signature or fingerprint) from all participants recruited. If any changes are made to the clinical research protocol, ICF, etc., the applicant will submit to the ethics committee for approval before implementation, and all changes will be fully documented. The results of this study will be made public on ClinicalTrial.gov and published in an open access medical journal after the study has ended. In addition, we will share the original data through the EDC system of Jiangsu Province Hospital of Chinese Medicine (http:// jshtcm.ecrfdata.com/edc/inx/jshtcm) or upon reasonable request to the corresponding author.

\section{CONCLUSIONS}

The AEEPN trial is a multicenter, prospective, randomized, SEA-controlled clinical trial that is powered to assess the efficacy and safety of EA on PHN and provide evidence-based medical evidence for EA treatment of PHN. The results of this study may help provide high-quality, up-todate evidence for patients, clinicians, and policy-makers seeking innovative and effective ways to treat PHN.

\section{Trial Status}

This study started recruiting on 14 October 2020 and is expected to be completed within 2 years. The first participant was recruited on 21 October 2020. At the time of this manuscript was written, a total of 158 participants have been recruited in the trial, and all seven participating clinical centers are actively recruiting, conducting follow-up visits, and collecting data.

\section{ACKNOWLEDGEMENTS}

We thank Suzhou Medical Supplies Factory Co., Ltd. for providing the ten SDZ-V electroacupuncture apparatuses and excellent technical assistance. We thank all study participants for their involvement in the study, and all the principal investigators and clinical staff participating in the trial for their efforts.

Funding. The study was funded by the Leading Talents Program of Traditional Chinese Medicine of Jiangsu Administration (Grant no. SLJ0206), and the Peak Academic Talents Program of Jiangsu Province Hospital of Chinese Medicine (Grant no. y2018rc05). The journal's Rapid Service Fee was funded by the Project of Building Evidence Based Practice Capacity for TCM of National Administration of Traditional Chinese Medicine 2019 (Grant no. 2019XZZXZJ008).

Authorship. All named authors meet the International Committee of Medical Journal Editors (ICMJE) criteria for authorship for this article, take responsibility for the integrity of the work as a whole, and have given their approval for this version to be published.

Authors' Contributions. Jianhua Sun, Qianyan Liu, and Jing Guo conceived and designed the AEEPN trial. Qianyan Liu, Xiaoliang $\mathrm{Wu}$, Jing Guo, and Bingyang Liu contributed to patient recruitment, clinical implementation, and/or data collection. Jianhua Sun, Lixia Pei, Qianyan Liu, Xiaoliang Wu, and Jie Gao provided technical or material support. Jianhua Sun obtained funding. Qianyan Liu, Jie Gao, Yuhang Wang, and Minghui Xia drafted the manuscript. Jianhua Sun and Lixia Pei critically revised the manuscript. Jianhua Sun and Lixia Pei supervised the study. All authors read and approved the final manuscript.

Disclosures. Qianyan Liu, Xiaoliang $\mathrm{Wu}$, Jing Guo, Jie Gao, Bingyang Liu, Yuhang Wang, Minghui Xia, Lixia Pei, and Jianhua Sun declare that they have no financial or other conflict of interest. 
Compliance with Ethics Guidelines. The AEEPN trial will be conducted in accordance with the principles of the Declaration of Helsinki, the Quality Management Practices for Clinical Trials of Medical Devices, and International Code of Ethics for Research on Human Health. It has been reviewed and approved by the Ethics Committee of all study centers (Jiangsu Province Hospital of Chinese Medicine [Primary sponsor]; Approval No. 2020NL103-03), Nanjing Hospital of Chinese Medicine Affiliated to Nanjing University of Chinese Medicine (Endorsed and shared the ethics approval documents of the primary sponsor), Wuxi Hospital of Traditional Chinese Medicine (Approval No. HX2020082402), Nantong Hospital of Traditional Chinese Medicine (Approval No. [2020] 008), Shuyang Hospital of Traditional Chinese Medicine (Approval No. 20200824-001), Huai'an Hospital of Traditional Chinese Medicine (Endorsed and shared the ethics approval documents of the primary sponsor), and Lianyungang Hospital of Traditional Chinese Medicine (Approval No. 2021-KY-05). The researchers will follow GCP principles and the approved protocol to implement clinical research and protect the health and rights of each patient. Consent to participate in the study will be obtained using an written informed consent (signature or fingerprint) from all participants recruited.

Data Availability. The data of this study are available from the corresponding author upon reasonable request.

Open Access. This article is licensed under a Creative Commons Attribution-NonCommercial 4.0 International License, which permits any non-commercial use, sharing, adaptation, distribution and reproduction in any medium or format, as long as you give appropriate credit to the original author(s) and the source, provide a link to the Creative Commons licence, and indicate if changes were made. The images or other third party material in this article are included in the article's Creative Commons licence, unless indicated otherwise in a credit line to the material. If material is not included in the article's Creative Commons licence and your intended use is not permitted by statutory regulation or exceeds the permitted use, you will need to obtain permission directly from the copyright holder. To view a copy of this licence, visit http://creativecommons.org/licenses/by$\mathrm{nc} / 4.0 /$.

\section{REFERENCES}

1. Johnson RW, Rice AS. Clinical practice. Postherpetic neuralgia. N Engl J Med. 2014;371(16): $1526-33$.

2. van Hecke O, Austin SK, Khan RA, Smith BH, Torrance N. Neuropathic pain in the general population: a systematic review of epidemiological studies. Pain. 2014;155(4):654-62.

3. Guthmiller CGKB. Postherpetic neuralgia. StatPearls. 2020. https://www.ncbi.nlm.nih.gov/books/ NBK493198/.

4. Cohen JI. Clinical practice: Herpes zoster. N Engl J Med. 2013;369(3):255-63.

5. John AR, Canaday DH. Herpes zoster in the older adult. Infect Dis Clin North Am. 2017;31(4): 811-26.

6. Werner RN, Nikkels AF, Marinovic B, et al. European consensus-based (S2k) guideline on the management of Herpes Zoster-guided by the European Dermatology Forum (EDF) in cooperation with the European Academy of Dermatology and Venereology (EADV), part 2: treatment. J Eur Acad Dermatol Venereol. 2017;31(1):20-9.

7. Johnson RW. Herpes zoster and postherpetic neuralgia. Expert Rev Vaccines. 2010;9(3 Suppl.):21-6.

8. Devor M. Rethinking the causes of pain in herpes zoster and postherpetic neuralgia: the ectopic pacemaker hypothesis. Pain Rep. 2018;3(6):e702.

9. Putri Mellaratna W, Jusuf NK, Yosi A. The impact of pain intensity on quality of life of postherpetic neuralgia patients. Med Glas (Zenica). 2020;17(2): 439-44.

10. Wu CHLZ, Zhao Y, Gao Y, et al. Electroacupuncture improves thermal and mechanical sensitivities in a rat model of postherpetic neuralgia. Mol Pain. 2013;9:18.

11. Li W, Peng W, Zhou J, Liu Z. Acupuncture for postherpetic neuralgia: a systematic review protocol. BMJ Open. 2014;4(11):e005725. 
12. Coyle ME, Liang $\mathrm{H}$, Wang $\mathrm{K}$, et al. Acupuncture plus moxibustion for herpes zoster: a systematic review and meta-analysis of randomized controlled trials. Dermatol Ther. 2017;30(4). https://doi.org/10. 1111/dth.12468.

13. Chan AW, Tetzlaff JM, Altman DG, et al. SPIRIT 2013 statement: defining standard protocol items for clinical trials. Ann Intern Med. 2013;158(3): 200-7.

14. Chan AW, Tetzlaff JM, Gotzsche PC, et al. SPIRIT 2013 explanation and elaboration: guidance for protocols of clinical trials. BMJ. 2013;346:e7586.

15. Agha RA, Altman DG, Rosin D. The SPIRIT 2013 statement-defining standard protocol items for trials. Int J Surg. 2015;13:288-91.

16. Pinnock H, Barwick M, Carpenter CR, et al. Standards for Reporting Implementation Studies (StaRI): explanation and elaboration document. BMJ Open. 2017;7(4):e013318.

17. Werner RN, Nikkels AF, Marinovic B, et al. European consensus-based ( $\mathrm{S} 2 \mathrm{k}$ ) guideline on the management of Herpes Zoster-guided by the European Dermatology Forum (EDF) in cooperation with the European Academy of Dermatology and Venereology (EADV), part 1: diagnosis. J Eur Acad Dermatol Venereol. 2017;31(1):9-19.

18. Gross GE, Eisert L, Doerr HW, et al. S2k guidelines for the diagnosis and treatment of herpes zoster and postherpetic neuralgia. J Dtsch Dermatol Ges. 2020;18(1):55-78.

19. Hjermstad MJ, Fayers PM, Haugen DF, et al. Studies comparing Numerical Rating Scales, Verbal Rating Scales, and Visual Analogue Scales for assessment of pain intensity in adults: a systematic literature review. J Pain Symptom Manag. 2011;41(6): 1073-93.

20. Hawker GA, Mian S, Kendzerska T, French M. Measures of adult pain: Visual Analog Scale for Pain (VAS Pain), Numeric Rating Scale for Pain (NRS Pain), McGill Pain Questionnaire (MPQ), ShortForm McGill Pain Questionnaire (SF-MPQ), Chronic Pain Grade Scale (CPGS), Short Form-36 Bodily Pain Scale (SF-36 BPS), and Measure of Intermittent and Constant Osteoarthritis Pain (ICOAP). Arthritis Care Res (Hoboken). 2011;63(Suppl 11):S240-52.

21. Ferreira-Valente MA, Pais-Ribeiro JL, Jensen MP. Validity of four pain intensity rating scales. Pain. 2011;152(10):2399-404.

22. Yamanishi R, Uchino M, Kawashima M, Dogru M, Matsuguma S, Tsubota K. Analysis of the association between the severity of ocular and systemic pain. Ocul Surf. 2019;17(3):434-9.

23. Chu LF, Cun T, Ngai LK, et al. Modulation of remifentanil-induced postinfusion hyperalgesia by the beta-blocker propranolol in humans. Pain. 2012;153(5):974-81.

24. Breen SP, Etter NM, Ziegler GR, Hayes JE. Oral somatosensatory acuity is related to particle size perception in chocolate. Sci Rep. 2019;9(1):7437.

25. Pickering G, Voute M, Macian N, Ganry H, Pereira B. Effectiveness and safety of 5\% lidocaine-medicated plaster on localized neuropathic pain after knee surgery: a randomized, double-blind controlled trial. Pain. 2019;160(5):1186-95.

26. Song JW, Lee YW, Yoon KB, Park SJ, Shim YH. Magnesium sulfate prevents remifentanil-induced postoperative hyperalgesia in patients undergoing thyroidectomy. Anesth Analg. 2011;113(2):390-7.

27. Martin E, Narjoz C, Decleves $X$, et al. Dextromethorphan analgesia in a human experimental model of hyperalgesia. Anesthesiology. 2019;131(2):356-68.

28. Mosteller RD. Simplified calculation of body-surface area. N Engl J Med. 1987;317(17):1098.

29. Redlarski G, Palkowski A, Krawczuk M. Body surface area formulae: an alarming ambiguity. Sci Rep. 2016;6:27966.

30. Mance M, Prutki M, Dujmovic A, Milosevic M, Vrbanovic-Mijatovic V, Mijatovic D. Changes in total body surface area and the distribution of skin surfaces in relation to body mass index. Burns. 2020;46(4):868-75.

31. Maarbjerg S, Gozalov A, Olesen J, Bendtsen L. Trigeminal neuralgia-a prospective systematic study of clinical characteristics in 158 patients. Headache. 2014;54(10):1574-82.

32. Dworkin RH, Turk DC, Trudeau JJ, et al. Validation of the Short-form McGill Pain Questionnaire-2 (SFMPQ-2) in acute low back pain. J Pain. 2015;16(4): $357-66$.

33. Buppha P, Tontisirin N. Reliability and validity of the Thai Short-Form McGill Pain Questionnaire-2 (SF-MPQ-2). J Anesth Clin Res. 2016;7(8). https:// doi.org/10.4172/2155-6148.1000650.

34. Lovejoy TI, Turk DC, Morasco BJ. Evaluation of the psychometric properties of the revised short-form McGill Pain Questionnaire. J Pain. 2012;13(12): 1250-7. 
35. Gauthier LR, Young A, Dworkin RH, et al. Validation of the short-form McGill pain questionnaire-2 in younger and older people with cancer pain. J Pain. 2014;15(7):756-70.

36. Maruo TNA, Maeda L, Shi K, T et al. Validity, reliability, and assessment sensitivity of the Japanese version of the short-form McGill pain questionnaire 2 in Japanese patients with neuropathic and nonneuropathic pain. Pain Med. 2014;15:1930-7.

37. Dworkin RH, Turk DC, Revicki DA, et al. Development and initial validation of an expanded and revised version of the Short-form McGill Pain Questionnaire (SF-MPQ-2). Pain. 2009;144(1-2): 35-42.

38. Coplan PM, Schmader K, Nikas A, et al. Development of a measure of the burden of pain due to herpes zoster and postherpetic neuralgia for prevention trials: adaptation of the brief pain inventory. J Pain. 2004;5(6):344-56.

39. Pincus T, Williams AC, Vogel S, Field A. The development and testing of the depression, anxiety, and positive outlook scale (DAPOS). Pain. 2004;109(1-2):181-8.

40. Rice ASC, Dworkin RH, McCarthy TD, et al. EMA401, an orally administered highly selective angiotensin II type 2 receptor antagonist, as a novel treatment for postherpetic neuralgia: a randomised, double-blind, placebo-controlled phase 2 clinical trial. Lancet. 2014;383(9929):1637-47.

41. Shkirkova K, Schuberg S, Balouzian E, et al. Paramedic global impression of change during prehospital evaluation and transport for acute stroke. Stroke. 2020;51(3):784-91.

42. Mentz RJ, Hernandez AF, Berdan LG, et al. Good clinical practice guidance and pragmatic clinical trials: balancing the best of both worlds. Circulation. 2016;133(9):872-80.
43. Hershman DL, Unger JM, Greenlee H, et al. Effect of acupuncture vs sham acupuncture or waitlist control on joint pain related to aromatase inhibitors among women with early-stage breast cancer: a randomized clinical trial. JAMA. 2018;320(2): 167-76.

44. He H. Treating chronic knee pain with acupuncture. JAMA. 2015;313:6.

45. Vickers AJ, Linde K. Acupuncture for chronic pain. JAMA. 2014;311(9):955-6.

46. Vickers AJ, Cronin AM, Maschino AC, et al. Acupuncture for chronic pain: individual patient data meta-analysis. Arch Intern Med. 2012;172(19): 1444-53.

47. Linde KSA, Jürgens S, Hoppe A, et al. Acupuncture for patients with migraine: a randomized controlled trial. JAMA. 2005;293(17):2118-25.

48. Zhao L, Li D, Zheng $\mathrm{H}$, et al. Acupuncture as adjunctive therapy for chronic stable angina: a randomized clinical trial. JAMA Intern Med. 2019;179(10):1388-97.

49. Marchant J. Acupuncture in cancer study reignites debate about controversial technique. Nature. 2017;552(7684):157-8.

50. Yang JW, Wang LQ, Zou X, et al. Effect of acupuncture for postprandial distress syndrome: a randomized clinical trial. Ann Intern Med. 2020;172(12):777-85.

51. Wu XK, Stener-Victorin E, Kuang HY, et al. Effect of acupuncture and clomiphene in chinese women with polycystic ovary syndrome: a randomized clinical trial. JAMA. 2017;317(24):2502-14. 